\title{
On a competitive system under chemotactic effects with non-local terms
}

\author{
Mihaela Negreanu and J Ignacio Tello
}

\begin{abstract}
In this paper, we study a system of partial differential equations describing the evolution of a population under chemotactic effects with non-local reaction terms. We consider an external application of chemoattractant in the system and study the cases of one and two populations in competition. By introducing global competitive/cooperative factors in terms of the total mass of the populations, we obtain, for a range of parameters, that any solution with positive and bounded initial data converges to a spatially homogeneous state with positive components. The proofs rely on the maximum principle for spatially homogeneous sub- and super-solutions.
\end{abstract}

\section{Introduction}

Chemotaxis is a biological process through which living organisms orient their movement along a chemical concentration gradient. The process is present in different types of biological phenomena such as bacteria aggregation, immune system response or angiogenesis in the embryo formation and in tumour development. Mathematical models to describe chemotaxis have been proposed in the last few years following the pioneering work of Keller and Segel [17] during the 1970s. Systems with chemotactic terms have been used to model not only the mentioned biological processes at microscopic scale but also population dynamics at macroscopic scale in the context of life sciences, 'gravitational collapse' in astrophysics, material sciences, etc. Several authors have studied the qualitative properties of these mathematical models to analyse global existence, pattern formation, finite-time blow-up, stability, etc. 
From a mathematical point of view, the Keller-Segel systems for one species and one chemoattractant can be classified depending on the type of differential equations involved in the model. Three main groups of systems have been studied: parabolic-parabolic systems, parabolic-elliptic and parabolic-ODE systems depending on the nature of the equation satisfied by the chemoattractant. Note that other types of systems also appear in the literature, with systems involving hyperbolic equations among them.

In this paper, we extend the Parabolic-Elliptic Keller-Segel system by introducing nonlocal terms in the logistic growth factor. One of the first mathematical studies for parabolicelliptic systems is the work by Jäger and Luckhaus [16], where a sub- and super-solutions method is applied to obtain finite-time blow-up in a two-dimensional domain. After [16], many authors have studied the question of blow-up for parabolic-elliptic chemotaxis systems, see for instance Nagai [22], Herrero et al [11], Biler [3] and the references therein for more details.

The problems studied in this paper present global existence of solutions produced by the logistic growth, which counteracts the blow-up tendency produced by chemotaxis. We denote by ' $u$ ' the density of the population of living organisms which satisfies a parabolic equation with constant diffusion and constant chemotactic sensitivity ' $\chi$ '. The equation presents a growth factor of logistic type defined in terms of the total mass of the population (the non-local term). If we denote the chemoattractant substance by ' $w$ ', the equation is as follows:

$$
u_{t}-\Delta u=-\chi \operatorname{div}(u \nabla w)+u\left(a_{0}-a_{1} u-\frac{a_{2}}{|\Omega|} \int_{\Omega} u\right), \quad x \in \Omega, t>0,
$$

for $a_{0}$ and $a_{1}$ positive, $a_{2} \in \mathbb{R}$, with Neumann boundary conditions and regular initial data. The logistic growth describes the competition of the individuals of the species for the resources of the environment and the cooperation to survive. The coefficient ' $a_{0}$ ', sometimes also called Malthusian parameter, induces an exponential growth for low density populations. At the time that the population grows, the competitive effect of the local term $a_{1} u$ becomes more influential. The non-local term $\left(a_{2} /|\Omega|\right) \int_{\Omega} u$ describes the influence of the total mass of the species in the growth of the population. If $a_{2}>0$, we have a competitive term which limits such growth and for $a_{2}<0$ the individuals cooperate globally to survive. In the last case, the individuals compete locally but cooperate globally and the effects of $a_{1} u$ and $\left(a_{2} /|\Omega|\right) \int_{\Omega} u$ balance the system. For $a_{2}<-a_{1}<0$ a blow-up may occur and for the case $a_{1}-\left(a_{2}\right)_{-}-\chi>0$ the solution exists globally in time.

In order to put our results in perspective, we recall various borderline cases. In particular, quite a number of works deal with the one-species system having $a_{2}=0$, which describes the situation where the influence of the non-local terms is neglected. This case has been studied in [26], where the assumption $a_{1}>2 \chi$ ensures the global stability of the homogeneous steady state. The parabolic-parabolic problem with logistic growth has also been studied in Hillen and Painter [12], where modelling details and numerical simulations are presented (see also references therein for further research). In Winkler [30], the blow-up of solutions is studied for a weak logistic growth term for a parabolic-elliptic system. In the limit case $\chi \rightarrow 0$ the system becomes a single reaction-diffusion equation modelling the evolution of a population where the motility is only produced by the diffusion. These types of equations have been studied in the generalized framework of the parabolic equation with non-local terms. See for instance [24] chapter $V$ and [18] (where $\Omega=\mathbb{R}$ ) and references therein for more details on that borderline case.

Logistic growth described by non-local terms has already been used in the context of chemotaxis by other authors. For instance, in [25] the authors suggest a growth coefficient rate in a competitive system modelling cancer cells behaviour which considers the influence 
of the surrounding area of a cell to replicate itself. The coefficient in [25] is given in the form

$$
\mu_{1}\left(1-\int_{\Omega} k_{1,1}(x, y) u(y) \mathrm{d} y-\int_{\Omega} k_{1,2}(x, y) v(y) \mathrm{d} y\right)
$$

where ' $u$ ' and ' $v$ ' denote the cancer cells density and the extracellular matrix density, respectively. The non-local term in [25] describes the 'competition' for the space between ' $u$ ' and ' $v$ '. Non-local terms of integral type also appear in the literature describing chemoattractant behaviour, see for instance [16]. There exists a wide literature studying one-species chemotaxis systems, we refer the reader to Horstmann [13] for a general overview of the subject.

In numerous biologically relevant processes, the latter signal substance is produced by the cells themselves and then its evolution is essentially governed by a parabolic equation of the form

$$
\epsilon w_{t}=d_{w} \Delta w+\tilde{f}(u, w)
$$

with positive constants $\epsilon$ and $d_{w}$ and a production term $\tilde{f}$ which, in extension to the situation in the classical approach, depends linearly on $u$ and $w$. Under the additional assumption that chemicals diffuse significantly faster than cells, when the degradation effects of the chemical are considered linear, a commonly used mathematically convenient simplification of previous equation is given by

$$
0=d_{w} \Delta w-\lambda w+\tilde{f}(u),
$$

under Neumann boundary conditions. In our model, the above elliptic approximation equation for $w$ is studied for the case where the chemical is also introduced in the system from outside, i.e.

$$
\tilde{f}(u)=u+f
$$

Therefore we may assume that the chemoattractant concentration ' $w$ ' satisfies a second-order elliptic equation of constant coefficients and the problem is described by the following system of partial differential equations:

$$
\left\{\begin{array}{l}
u_{t}-\Delta u=-\chi \operatorname{div}(u \nabla w)+u\left(a_{0}-a_{1} u-a_{2} \int_{\Omega} u\right) \\
-\Delta w+\lambda w=f+u, \quad x \in \Omega, t>0 \\
\frac{\partial u}{\partial n}=\frac{\partial w}{\partial n}=0, x \in \partial \Omega, t>0 \\
\left.u\right|_{t=0}=u_{0} .
\end{array}\right.
$$

Biological systems in experimental environments are frequently acted on by an artificial external chemical force. The effects of a direct application of a chemoattractant have also been considered in the literature (see for instance [21]), where the external flux of a chemical substance is introduced through the boundary to control the pattern formation. In [27], an application in the interior of the domain is considered in terms of Dirac functions. External forces applied in the system, at the interior or through the boundary, provide the possibility to guide the system towards a desired state.

In this paper, we consider the case where the living organisms are divided into two subspecies ' $u$ ' and ' $v$ ' attracted chemotactically by the same signal substance. Both subspecies diffuse with constant diffusion coefficient (assumed 1 for both subspecies) and with different chemotactic coefficients ' $\chi_{1}$ ' and ' $\chi_{2}$ '. The growth terms are given, as in the previous case, as a logistic growth with integral terms of the form

$u\left(a_{0}-a_{1} u-a_{2} v-a_{3} \int_{\Omega} u-a_{4} \int_{\Omega} v\right) \quad$ and $\quad v\left(b_{0}-b_{1} u-b_{2} v-b_{3} \int_{\Omega} u-b_{4} \int_{\Omega} v\right)$, respectively. 
The sign of the coefficients represents local competition if $a_{1}, a_{2}, b_{1}$ and $b_{2}$ are positive. The subspecies globally compete if $a_{4}$ and $b_{3}$ are positive and globally cooperate if both are negative. The coefficients $a_{3}$ and $b_{4}$ describe the global influence of each subspecies in its population. As before, the chemical concentration is produced by both subspecies at a constant rate and is introduced artificially by an external application. The problem of two species is described by the following system:

$$
\left\{\begin{array}{l}
u_{t}-\Delta u=-\chi_{1} \nabla \cdot(u \nabla w)+u\left(a_{0}-a_{1} u-a_{2} v-a_{3} \int_{\Omega} u-a_{4} \int_{\Omega} v\right), \\
v_{t}-\Delta v=-\chi_{2} \nabla \cdot(v \nabla w)+v\left(b_{0}-b_{1} u-b_{2} v-b_{3} \int_{\Omega} u-b_{4} \int_{\Omega} v\right), \\
-\Delta w+\lambda w=f+k_{1} u+k_{2} v,
\end{array}\right.
$$

with the homogeneous Neumann boundary conditions

$$
\frac{\partial u}{\partial v}=\frac{\partial v}{\partial v}=\frac{\partial w}{\partial v}=0, \quad x \in \partial \Omega, \quad t>0,
$$

and initial data

$$
u(x, 0)=u_{0}(x), \quad v(x, 0)=v_{0}(x), \quad x \in \Omega .
$$

Motivated by biological experiments (see for instance Lauffenburger [20]), multi-species chemotaxis systems become a rich mathematical problem studied by several authors. As in the one-species problem, the finite-time blow-up/global existence question is an interesting mathematical challenge with biological implications. In [10] the authors studied the existence of non-trivial equilibrium solutions via bifurcation methods for a two biological species with two chemical agents. Systems of two biological species and one common attractor without logistic growth factors have been studied in several works describing the blow-up phenomenon, see [7-9] for results in bounded domains. Conca and Espejo [5, 6] studied the two-dimensional case in the whole space. In Horstmann [14], a general situation for the multi-species chemotaxis model in the presence of one or several chemical stimuli is treated. See also Kuiper [19] and Wang and Wu [29].

We assume throughout the paper that the forcing term $f$ is uniformly bounded and $f \in C_{x, t}^{\alpha, \beta}(\bar{\Omega} \times[0, \infty))$ for $\alpha>0$ and $\beta \geqslant 1+\frac{\alpha}{2}$. For the first problem, one of the following assumptions is required to study the asymptotic stability of the solutions:

or

$$
\left\|f-\frac{1}{|\Omega|} \int_{\Omega} f\right\|_{L^{\infty}(\Omega)} \longrightarrow 0, \quad \text { as } \quad t \rightarrow \infty
$$

$$
\int_{0}^{\infty}\left|\sup _{x \in \Omega} f-\inf _{x \in \Omega} f\right| \leqslant C_{0}<\infty .
$$

Note that $(0.5)$ is equivalent to

$$
\left|\sup _{x \in \Omega} f-\inf _{x \in \Omega} f\right|_{L^{\infty}(\Omega)} \longrightarrow 0, \quad \text { as } \quad t \rightarrow \infty .
$$

The second problem presents some differences compared with the single-species system and only under the second assumption (0.6) do we obtain the desired result. More details about the choice of hypothesis (0.5) or (0.6) are given at the end of this section and in the conclusions.

In this paper, we consider $\Omega \subset \mathbb{R}^{n}$, for $n \geqslant 1$, a bounded domain with a smooth boundary $\partial \Omega$ and, for simplicity, we take

$$
|\Omega|=1 .
$$


The main results of the paper are stated below:

Theorem 0.1. For every $a_{0}>0, a_{1}>0, \chi>0, a_{2} \in \mathbb{R}$, verifying

$$
a_{1}>2 \chi+\left|a_{2}\right|
$$

and $f$ satisfying either $(0.5)$ or $(0.6)$, for positive data $u_{0} \in C_{x}^{\alpha}(\bar{\Omega})$ satisfying the boundary condition $\left(\partial u_{0} / \partial n\right)=0$, the solution to $(0.1)$ fulfills

$$
\lim _{t \rightarrow \infty}\left\|u-u^{*}\right\|_{L^{\infty}(\Omega)}=0
$$

where

$$
u^{*}=\frac{a_{0}}{a_{1}+a_{2}} .
$$

In the case of two subspecies we work under the following assumptions:

$\chi_{1}, \chi_{2}, k_{1}, k_{2}, a_{i}, b_{i}>0, \quad$ for $i=1,2$,

$a_{i} \in \mathbb{R}, b_{i} \in \mathbb{R}, \quad$ for $i=3,4$

$a_{1}>2 k_{1}\left(\chi_{1}+\chi_{2}\right)+b_{1}+\left|b_{3}\right|+\left|a_{3}\right| \quad$ and $\quad b_{2}>2 k_{2}\left(\chi_{1}+\chi_{2}\right)+a_{1}+\left|a_{4}\right|+\left|b_{4}\right|$.

In this second case we study the behaviour of the solutions, which tend to the constants

$$
u^{*} \equiv \frac{a_{0}\left(b_{2}+b_{4}\right)-b_{0}\left(a_{2}+a_{4}\right)}{\left(b_{2}+b_{4}\right)\left(a_{1}+a_{3}\right)-\left(b_{1}+b_{3}\right)\left(a_{2}+a_{4}\right)}
$$

and

$$
v^{*} \equiv \frac{a_{0}\left(b_{1}+b_{3}\right)-b_{0}\left(a_{1}+a_{3}\right)}{\left(b_{1}+b_{3}\right)\left(a_{2}+a_{4}\right)-\left(b_{2}+b_{4}\right)\left(a_{1}+a_{3}\right)} .
$$

The main result, given in section 2 , is as follows:

Theorem 0.2. Assume that (0.6), (0.9)-(0.11) hold. Then, for all positive initial data $u_{0}$, $v_{0} \in C_{x}^{\alpha}(\bar{\Omega})$ satisfying the boundary condition $\left(\partial u_{0} / \partial n\right)=\left(\partial v_{0} / \partial n\right)=0$, the solution $(u, v)$ to $(0.2)$ is bounded and satisfies

$$
\left\|u(\cdot, t)-u^{*}\right\|_{L^{\infty}(\Omega)}+\left\|v(\cdot, t)-v^{*}\right\|_{L^{\infty}(\Omega)} \rightarrow 0, \quad \text { as } t \rightarrow \infty .
$$

The proofs of theorems 0.1 and 0.2 follow a comparison argument based on upper and lower solutions defined by ordinary systems of differential equations. Similar comparison arguments have been used in the context of chemotaxis in other papers, see for instance [26,28] or [23]. In [23], the method is applied to a case of non-constant chemotaxis coefficient, defined by $\chi(u)=\chi_{0}(N-u)$, which becomes negative for a large concentration of $u$. The method can also be applied to more general parabolic-elliptic chemotaxis systems with reaction terms.

The assumptions required for $f$ in theorem 0.1 are more general than those that we assume in theorem 0.2. They are introduced in order to proof the asymptotic behaviour of the solutions in the associated ODEs system. System (0.1) is a non-autonomous Lotka-Volterra competition system of two equations. These types of systems are well studied in the literature (see for instance $[1,2]$ and references therein for details) while $(0.2)$ contains competitive and cooperative terms which make the problem more difficult. For that reason, we detail the proof of lemma 2.2, where the asymptotic stability of the associated ODEs system is studied.

In light of known research in the corresponding borderline case, it seems natural to conjecture that the dampening effect of the non-local terms (for instance, on the corresponding single-species system, when $a_{2}$ is a large positive number) might lead to an even more effective homogenization. 
As we shall see in the following sections, we prove the asymptotic behaviour of the solutions by comparison with solutions of ordinary differential equations. The results are presented under hypotheses for the coefficients equations which essentially reflect the assumption that the effects stemming from chemotactic cross-diffusion and competitive degradation are sufficiently small.

Thanks to the comparison method, we have that, for every $a_{1}$ and $a_{2}$ positive and large enough, checking the necessary relation $a_{1}>2 \chi+a_{2}$, the results are still valid. If this condition fails, sometimes it is referred to as the principle of competitive exclusion, i.e. there can be no coexistence of the two species $u$ and $\bar{u}$, one of them will be driven to extinction while the other will stabilize at a certain solution of a logistic equation. This case only provides us with information about the boundedness of the solution $u$ of $(0.1)$ and no information about the asymptotic behaviour.

A deeper insight can be expected here upon addressing the corresponding mathematical issues of instability of constant steady states, or existence of non-constant equilibrium. But these require entirely different approaches than pursued here, and thus need to be discussed elsewhere. A similar comment applies to the two-species system.

\section{One-species chemotaxis system under assumption $(0.5)$}

In [26], the authors have considered the case $f=0$ and $a_{2}=0$, under assumption $a_{1}>2 \chi$. The system possesses a uniquely determined spatially homogeneous positive equilibrium $u^{*}$, globally asymptotically stable within a certain non-empty range of the logistic growth coefficients.

If we denote by $v:=w-F$, with $F$ solution of $-\Delta F+\lambda F=f$, system (0.1) becomes

$$
\left\{\begin{array}{l}
u_{t}=\Delta u-\chi \nabla u[\nabla v+\nabla F]+u\left(\chi(u-\lambda v+f-\lambda F)+a_{0}-a_{1} u-a_{2} \int_{\Omega} u\right), \\
-\Delta v+\lambda v=u, \quad x \in \Omega, t>0, \\
-\Delta F+\lambda F=f, \quad x \in \Omega, t>0, \\
\frac{\partial u}{\partial n}=\frac{\partial v}{\partial n}=0, \quad x \in \partial \Omega, \quad t>0, \\
\left.u\right|_{t=0}=u_{0} .
\end{array}\right.
$$

Since $u$ and $v$ represent densities, the solutions of $(0.1)$, which are biologically meaningful, must satisfy

$$
u \geqslant 0 \quad \text { and } \quad v \geqslant 0 \text {. }
$$

Thus, it is reasonable to require throughout that the initial data $u_{0} \in C_{x}^{\alpha}(\bar{\Omega})$ be non-negative. As a preliminary, let us state the following result on existence and uniqueness of solutions:

Lemma 1.1. Let $\alpha \in(0,1)$ and $u_{0} \in C_{x}^{\alpha}(\bar{\Omega})$ a non-negative initial data satisfying the boundary condition $\partial u_{0} / \partial n=0$. Then, there exists $T_{\max } \in(0, \infty]$ and a unique pair $(u, v)$ of positive functions

$$
\begin{aligned}
& u \in C_{x, t}^{2+\alpha, 1+\frac{\alpha}{2}}\left(\bar{\Omega} \times\left(0, T_{\max }\right)\right), \\
& v \in C_{x, t}^{2+\alpha, 1+\frac{\alpha}{2}}\left(\bar{\Omega} \times\left(0, T_{\max }\right)\right)
\end{aligned}
$$

such that $(u, v)$ is a classical solution of $(0.1)$ in $\Omega \times\left(0, T_{\max }\right)$. Moreover, if $T_{\max }<\infty$, then

$$
\lim _{t \rightarrow T_{\max }}\|u(t)\|_{L^{\infty}(\Omega)}=\infty .
$$


Proof. The proof of the lemma is similar to the proof of theorem 2.1 in [26] and is a straightforward adaptation of well-established methods based on standard arguments (see [15], for instance).

We now introduce the standard notation for positive and negative part functions which we shall use in several proofs along the paper:

$$
(s)_{+}=\left\{\begin{array}{l}
s \text { if } s \geqslant 0 \\
00 \text { otherwise }
\end{array} \quad(s)_{-}=(-s)_{+} .\right.
$$

Lemma 1.2. Under the same hypotheses than previous lemma, problem (0.1) possesses a unique and uniformly bounded global classical solution $(u, v)$. More precisely, there exists $c=c\left(\left\|u_{0}\right\|_{L^{\infty}(\Omega)}\right)$ such that

$$
\|u(t)\|_{L^{\infty}(\Omega)} \leqslant c\left(\left\|u_{0}\right\|_{L^{\infty}(\Omega)}\right), \quad \forall t \in(0, \infty)
$$

holds.

Proof. We first consider the solution to the following logistic equation:

$$
\begin{aligned}
& \tilde{u}_{t}=\tilde{u}\left(2 \chi\|f\|_{L^{\infty}\left(\Omega_{T}\right)}+a_{0}-\left(a_{1}-\left(a_{2}\right)_{-}-\chi\right) \tilde{u}\right), \quad t \in(0, T) \\
& \tilde{u}_{0}=\left\|u_{0}\right\|_{L^{\infty}(\Omega)},
\end{aligned}
$$

for $T<T_{\max }$ and $\Omega_{T}=\Omega \times[0, T]$. We consider the function $\tilde{U}=u-\tilde{u}$ which satisfies

$\tilde{U}_{t}-\Delta \tilde{U}=-\chi \nabla \tilde{U} \cdot \nabla v-\chi \nabla \tilde{U} \cdot \nabla F+\chi u(u-\lambda v)+\chi u(f-\lambda F)$

$$
+u\left(a_{0}-a_{1} u-a_{2} \int_{\Omega} u\right)-\tilde{u}\left(2 \chi\|f\|_{L^{\infty}}+a_{0}-\tilde{u}\left(a_{1}-\left(a_{2}\right)_{-}-\chi\right)\right) .
$$

Since

$$
|f-\lambda F| \leqslant 2\|f\|_{L^{\infty}\left(\Omega_{T}\right)} \quad \text { and } \quad v \geqslant 0
$$

we have

$$
\begin{aligned}
\tilde{U}_{t}-\Delta \tilde{U} \leqslant- & \chi \nabla \tilde{U} \cdot \nabla v-\chi \nabla \tilde{U} \cdot \nabla F+\left(\chi-a_{1}\right)\left(u^{2}-\tilde{u}^{2}\right)+\left(2 \chi\|f\|_{L^{\infty}\left(\Omega_{T}\right)}+a_{0}\right) \tilde{U} \\
& -a_{2} u \int_{\Omega} u-\left(a_{2}\right) \tilde{u}^{2} .
\end{aligned}
$$

- If $a_{2}>0$, by the mean value theorem, there exists

$$
\xi(x, t) \in(u(t, x), \tilde{u}(t)) \cup(\tilde{u}(t), u(t, x))
$$

such that

$$
\tilde{U}_{t}-\Delta \tilde{U} \leqslant-\chi \nabla \tilde{U} \cdot \nabla v-\chi \nabla \tilde{U} \cdot \nabla F+\left(2\left(\chi-a_{1}\right) \xi+\left(2 \chi\|f\|_{L^{\infty}\left(\Omega_{T}\right)}+a_{0}\right)\right) \tilde{U} .
$$

- If $a_{2}<0$, then $-\left(a_{2}\right)_{-}=a_{2}$ and

$$
\begin{array}{r}
-a_{2} u \int_{\Omega} u+a_{2} \tilde{u}^{2}=\left|a_{2}\right| \tilde{U} \int_{\Omega} u+\left|a_{2}\right| \tilde{u}\left(\int_{\Omega} u-\tilde{u}\right) \\
\leqslant\left|a_{2}\right| \tilde{U} \int_{\Omega} u+\left|a_{2}\right| \tilde{u} \int_{\Omega} \tilde{U} \leqslant\left|a_{2}\right| \tilde{U} \int_{\Omega} u+\left|a_{2}\right| \tilde{u} \int_{\Omega} \tilde{U}_{+} .
\end{array}
$$


We take $\tilde{U}_{+}$as test function, i.e. we multiply by $\tilde{U}_{+}$and we integrate by parts over $\Omega$, through the inequalities

$-\chi \int_{\Omega} \tilde{U}_{+} \nabla \tilde{U} \cdot \nabla F=-\frac{\chi}{2} \int_{\Omega} \nabla \tilde{U}_{+}^{2} \cdot \nabla F=\frac{\chi}{2} \int_{\Omega} \tilde{U}_{+}^{2}(\lambda F-f) \leqslant \chi\|f\|_{L^{\infty}(\Omega)} \int_{\Omega} \tilde{U}_{+}^{2}$,

$-\chi \int_{\Omega} \tilde{U}_{+} \nabla \tilde{U} \cdot \nabla v=-\frac{\chi}{2} \int_{\Omega} \nabla \tilde{U}_{+}^{2} \cdot \nabla v=\frac{\chi}{2} \int_{\Omega} \tilde{U}_{+}^{2}(-u+\lambda v) \leqslant \frac{\lambda \chi}{2}\|v\|_{L^{\infty}(\Omega)} \int_{\Omega} \tilde{U}_{+}^{2}$,

we obtain the following relation:

$$
\frac{1}{2} \frac{\mathrm{d}}{\mathrm{d} t} \int_{\Omega} \tilde{U}_{+}^{2}+\int_{\Omega}\left|\nabla \tilde{U}_{+}\right|^{2} \leqslant k\left(1+\|v\|_{L^{\infty}\left(\Omega_{T}\right)}\right)\left(\int_{\Omega} \tilde{U}_{+}^{2}+\left(\int_{\Omega} \tilde{U}_{+}\right)^{2}\right) .
$$

By Young's inequality, we have that

$$
\left(\int_{\Omega} \tilde{U}_{+}\right)^{2} \leqslant \int_{\Omega} \tilde{U}_{+}^{2}
$$

and therefore

$$
\frac{1}{2} \frac{\mathrm{d}}{\mathrm{d} t} \int_{\Omega} \tilde{U}_{+}^{2}+\leqslant 2 k\left(1+\|v\|_{L^{\infty}\left(\Omega_{T}\right)}\right) \int_{\Omega} \tilde{U}_{+}^{2}
$$

which give us $u \leqslant \tilde{u}$ in $(0, T)$. Taking limits when $T \rightarrow T_{\max }$, thanks to lemma 1.1 and the inequality

$$
\|v\|_{L^{\infty}(\Omega)} \leqslant \frac{1}{\lambda}\|u\|_{L^{\infty}(\Omega)}
$$

we conclude the proof of the lemma.

Remark 1.3. For $a_{2}<-a_{1}<0$ and $f=0$ we have that a finite-time blow-up occurs. The spatially homogeneous solution satisfies the ODE

$$
\frac{\partial u}{\partial t}=u\left(a_{0}-\left(a_{1}+a_{2}\right) u\right)
$$

where solutions blow up at finite time for any positive initial data.

\subsection{A priori estimates}

In order to study the large time behaviour of solutions of $(0.1)$ we consider the case

$$
a_{2}>0 \text {. }
$$

At the end of the following subsection we present a sketch of the proof for $a_{2}<0$.

If we denote by

$$
\bar{f}(t)=\sup _{x \in \Omega} f(t, x), \quad \underline{f}(t)=\inf _{x \in \Omega} f(t, x),
$$

by the maximum principle applied to $(1.1)$, we know that

$$
\underline{f}(t) \leqslant \lambda F(x, t) \leqslant \bar{f}(t) .
$$

By assumption (0.5), we have

$$
\lambda F(x, t) \rightarrow \frac{1}{|\Omega|} \int_{\Omega} f, \quad \text { as } t \rightarrow \infty, \quad \text { in } \quad L^{\infty}(\Omega)
$$

and therefore

$$
\sup _{x \in \Omega}\{f(x, t)-\lambda F(x, t)\} \rightarrow 0, \quad \inf _{x \in \Omega}\{f(x, t)-\lambda F(x, t)\} \rightarrow 0 .
$$


Note that, by integration in $-\Delta F+\lambda F=f$, we obtain that $\int_{\Omega}(f-\lambda F)=0$, for all $t>0$ which implies that $\sup _{x \in \Omega}\{f(x, t)-\lambda F\} \geqslant 0$ and $\inf _{x \in \Omega}\{f(x, t)-\lambda F\} \leqslant 0$. For simplicity, let us denote by

$$
M(t):=\chi(\bar{f}(t)-\underline{f}(t)), \quad \tilde{a}_{0}^{+}(t):=a_{0}+M(t) \quad \text { and } \quad \tilde{a}_{0}^{-}(t):=a_{0}-M(t) .
$$

We introduce a system of the initial-value problem for the upper and lower solutions, $(\bar{u}, \underline{u})=(\bar{u}(t), \underline{u}(t))$,

$$
\begin{cases}\bar{u}^{\prime}=\bar{u}\left[\tilde{a}_{0}^{+}(t)-\left(a_{1}-\chi\right) \bar{u}-\left(\chi+a_{2}\right) \underline{u}\right], & t>0, \\ \underline{u}^{\prime}=\underline{u}\left[\tilde{a}_{0}^{-}(t)-\left(\chi+a_{2}\right) \bar{u}-\left(a_{1}-\chi\right) \underline{u}\right], & t>0,\end{cases}
$$

with non-negative initial data

$$
\bar{u}(0)=\bar{u}_{0}, \quad \underline{u}(0)=\underline{u}_{0} \quad \text { and } \quad 0<\underline{u}_{0}<\bar{u}_{0} .
$$

Taking into account $(0.5)$ we have $\lim _{t \rightarrow \infty} \tilde{a}_{0}^{+}(t)=\lim _{t \rightarrow \infty} \tilde{a}_{0}^{-}(t)=a_{0}$ and we can choose the positive parameter $a_{0}$ such that

$$
\tilde{a}_{0}^{-}(t) \geqslant 0, \quad \forall t \geqslant t_{0},
$$

for $t_{0}$ large enough. Functions $\tilde{a}_{0}^{+}(t)$ and $\tilde{a}_{0}^{-}(t)$ are continuous and bounded above and below by positive constants, assumed $a_{1}>\chi$. This will be the framework for the rest of this section and all the results are valid under these conditions.

System (1.5) (commonly called a non-autonomous Lotka-Volterra system) as a model of competition between two species has been widely studied in the literature. For the autonomous case, i.e. $M(t)=0$, Braun (in [4]) details the asymptotic behaviour depending on the parameters. To the authors' knowledge, the asymptotic properties of the solutions of a general case of the non-autonomous system (1.5) was studied for the first time by Ahmad in [1], for bounded, continuous and non-negative coefficients. Using only simple arguments based on differential inequalities and standard theorems concerning the continuity of solutions of differential equations with respect to initial conditions and parameters, it is possible to find optimal bounds and convergence results for the solutions of (1.5).

For the reader's convenience we quote the results in [4] and [1] used in the proof of the main result concerning the ODEs system. Given a function $g(t)$, which is bounded above and below by positive constants for $t_{0} \leqslant t<\infty$, we let $g_{L}$ and $g_{M}$ denote inf $t_{t \geqslant t_{0}} g(t)$ and $\sup _{t \geqslant t_{0}} g(t)$, respectively. Recall that the coefficients in (1.5) are always assumed to be bounded, continuous and non-negative.

(i) $(\boldsymbol{M}=\mathbf{0})$ In this case we have an autonomous system (commonly called an autonomous Lotka-Volterra system) as a model of competition between two species

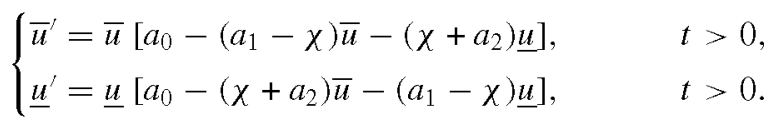

One of the equilibrium points of (1.8) is $u^{*}=\underline{u}^{*}=\bar{u}^{*}$ given by

$$
u^{*}:=\frac{a_{0}}{a_{1}+a_{2}} .
$$

In [4] and references therein, it is proved that a phase plane analysis of this autonomous case shows that the condition

$$
a_{1}>2 \chi+a_{2}
$$

is necessary and sufficient for the existence of a unique stable equilibrium point $\left(\bar{u}^{*}, \underline{u}^{*}\right) \neq$ $(0,0)$ of system (1.8) given by (1.9), such that both components are positive and it globally attracts all solutions with initial values in the open first quadrant of the $(\bar{u}, \underline{u})$ plane. 
(ii) $(\boldsymbol{M} \neq \mathbf{0})$ In [1] it was shown that if the coefficients $\tilde{a}_{0}^{ \pm}(\cdot)$ are bounded below and above by positive constants, and verify

$$
\tilde{a}_{0 L}^{+}>\frac{\left(\chi+a_{2}\right) \tilde{a}_{0 M}^{-}}{a_{1}-\chi} \quad \text { and } \quad \tilde{a}_{0 L}^{-}>\frac{\left(\chi+a_{2}\right) \tilde{a}_{0 M}^{+}}{a_{1}-\chi},
$$

then there exists a solution $u^{*}(t)=\left(\underline{u}^{*}(t), \bar{u}^{*}(t)\right)$ such that the inequalities

$$
\begin{aligned}
& \frac{\tilde{a}_{0 L}^{+}\left(a_{1}-\chi\right)-\left(\chi+a_{2}\right) \tilde{a}_{0 M}^{-}}{\left(a_{1}+a_{2}\right)\left(a_{1}-2 \chi-a_{2}\right)} \equiv s_{1} \leqslant \bar{u}^{*}(t) \leqslant r_{1} \equiv \frac{\tilde{a}_{0 M}^{+}\left(a_{1}-\chi\right)-\left(\chi+a_{2}\right) \tilde{a}_{0 L}^{-}}{\left(a_{1}+a_{2}\right)\left(a_{1}-2 \chi-a_{2}\right)} \\
& \frac{\tilde{a}_{0 L}^{-}\left(a_{1}-\chi\right)-\left(\chi+a_{2}\right) \tilde{a}_{0 M}^{+}}{\left(a_{1}+a_{2}\right)\left(a_{1}-2 \chi-a_{2}\right)} \equiv r_{2} \leqslant \underline{u}^{*}(t) \leqslant s_{2} \equiv \frac{\tilde{a}_{0 M}^{-}\left(a_{1}-\chi\right)-\left(\chi+a_{2}\right) \tilde{a}_{0 L}^{+}}{\left(a_{1}+a_{2}\right)\left(a_{1}-2 \chi-a_{2}\right)}
\end{aligned}
$$

hold, for $t_{0} \leqslant t<\infty$. These bounds are optimal in the sense that, in the autonomous problem, the upper bound for each component coincides with the lower bound for that component. Another important result obtained in [1] is: if conditions (1.11) hold and $\left(\bar{u}_{1}(t), \underline{u}_{1}(t)\right)$ and $\left(\bar{u}_{2}(t), \underline{u}_{2}(t)\right)$ are any two solutions of $(1.5)$ for positive initial data, then $\bar{u}_{1}(t)-\bar{u}_{2}(t) \rightarrow 0$ and $\underline{u}_{1}(t)-\underline{u}_{2}(t) \rightarrow 0$ as $t \rightarrow \infty$. Thus it follows that if $(\bar{u}(t), \underline{u}(t))$ is any solution of $(1.5)$ with both components positive at some time and $\varepsilon$ is any arbitrary positive number, then

$$
s_{1}-\varepsilon<\bar{u}(t)<r_{1}+\varepsilon, \quad r_{2}-\varepsilon<\underline{u}(t)<s_{2}+\varepsilon
$$

for sufficiently large $t$.

Moreover, if $\bar{u}(t)$ and $\underline{u}(t)$ are positive solutions of the logistic equations $\bar{u}^{\prime}(t)=$ $\bar{u}(t)\left[\tilde{a}_{0}^{+}(t)-\left(a_{1}-\chi\right) \bar{u}(t)\right]$ and $\underline{u}^{\prime}(t)=\underline{u}(t)\left[\tilde{a}_{0}^{-}(t)-\left(a_{1}-\chi\right) \underline{u}(t)\right]$, respectively, then the pairs $(\bar{u}(t), 0)$ and $(0, \underline{u}(t))$ are solutions of (1.5). Thus, it follows from the uniqueness theorem that the open first quadrant in the $(\bar{u}, \underline{u})$-plane is invariant in the sense that if $(\bar{u}(t), \underline{u}(t))$ is a solution of $(1.5)$ with $\bar{u}(\tilde{t})>0$ and $\underline{u}(\tilde{t})>0$ for some $\tilde{t}$ then $\bar{u}(t)>0$ and $\underline{u}(t)>0$ for all $t$ in the domain of $(\bar{u}(t), \underline{u}(t))$. Similarly, the first closed quadrant in the $(\bar{u}, \underline{u})$-plane is invariant.

Remark 1.4. Passing to the limit, $t \rightarrow \infty$, in inequalities (1.11), we find (1.10), i.e.

$$
a_{1}>2 \chi+a_{2},
$$

which is the equivalent condition found in [26] for the particular case $f=0$ and $a_{2}=0$.

For any positive parameters $\chi$ and $a_{i}$ (for $\left.i=0,1,2\right)$ under assumption (1.7) and (1.10) any solution $(\bar{u}(t), \underline{u}(t))$, of $(1.5)$, with positive initial data satisfies

$$
\lim _{t \rightarrow \infty} \bar{u}^{*}(t)=\lim _{t \rightarrow \infty} \underline{u}^{*}(t)=u^{*}=\frac{a_{0}}{a_{1}+a_{2}}
$$

for the particular case $f=0$. In other words, we have that all solutions $(\bar{u}(t), \underline{u}(t))$ of (1.5), with both pairs $\left(\bar{u}\left(t_{0}\right), \underline{u}\left(t_{0}\right)\right)$ positive, ultimately approach the equilibrium solution (1.9).

Remark 1.5. If one of the conditions (1.11) fails, sometimes it is referred to as the principle of competitive exclusion. An extension of this principle for non-autonomous systems was given in [2], where it was shown that similar algebraic inequalities imply that there can be no coexistence of the two species; one of them will be driven to extinction while the other will stabilize at a certain solution of a logistic equation. Hypothesis $(0.5)$ is essential and it is the key of the proof with this method. We do not give more details because this case only provides us with information about the boundedness of solution of $(0.1)$ and no information about the asymptotic behaviour. 


\subsection{Comparison Method}

According to the strong maximum principle (see [24, proposition $52.7 \mathrm{p} \mathrm{511])} \mathrm{applied} \mathrm{to} \mathrm{the}$ first equation in (0.1), replacing $t$ by $t+\tau$ for sufficiently small $\tau>0$ we may assume that

$$
u_{0}>0 \quad \text { in } \bar{\Omega} \text {. }
$$

It is therefore possible to find positive numbers $\bar{u}_{0}$ and $\underline{u}_{0}$ such that the inequalities

$$
0<\underline{u}_{0}<u^{*}=\frac{a_{0}}{a_{1}+a_{2}}<\bar{u}_{0}
$$

hold as well as

$$
\underline{u}_{0} \leqslant u_{0}(x) \leqslant \bar{u}_{0} \quad \text { for all } x \in \Omega .
$$

First of all we have the following properties of the solution $u$ of $(0.1)$.

Lemma 1.6. There exists $c=c\left(\left\|u_{0}\right\|_{L^{\infty}(\Omega)}\right)$ such that

$$
\int_{\Omega} u \leqslant c, \quad \forall t \in\left(0, T_{\max }\right)
$$

Proof. Integrating in space variable over $\Omega$ the first equation in $(0.1)$, we have

$$
\frac{\mathrm{d}}{\mathrm{d} t} \int_{\Omega} u=a_{0} \int_{\Omega} u-a_{1} \int_{\Omega} u^{2}-a_{2}\left(\int_{\Omega} u\right)^{2} .
$$

Thanks to Hölder inequality it results

$$
\frac{\mathrm{d}}{\mathrm{d} t} \int_{\Omega} u \leqslant a_{0} \int_{\Omega} u-\left(C_{1}(\Omega) a_{1}+a_{2}\right)\left(\int_{\Omega} u\right)^{2}
$$

which implies, solving the previous logistic equation, that

which ends the proof.

$$
\int_{\Omega} u \leqslant \max \left\{\int_{\Omega} u_{0}, \frac{a_{0}}{C_{1}(\Omega) a_{1}+a_{2}}\right\},
$$

Lemma 1.7. For any non-negative initial data $u_{0} \in C_{x}^{\alpha}(\bar{\Omega})$ (for $\alpha \in(0,1)$ ) the solution to (0.1) fulfills

$$
\underline{u} \leqslant u \leqslant \bar{u}
$$

Proof. We shall derive an appropriate differential inequality for some functional involving the functions $\bar{U}$ and $\underline{U}$ which are defined by setting

$$
\bar{U}(x, t):=u(x, t)-\bar{u}(x, t) \quad \text { and } \quad \underline{U}(x, t):=u(x, t)-\underline{u}(x, t) .
$$

In order to verify that the positive and negative parts $\bar{U}_{+}$and $\underline{U}_{-}$are identically zero throughout $\Omega \times\left(0, T_{\max }\right)$, we denote by $g$ the quadratic term $g(s):=s\left(a_{0}-a_{1} s\right)$. Then $\bar{U}$ satisfies $\bar{U}_{t}-\Delta \bar{U}=-\chi \nabla \bar{U} \cdot \nabla v-\chi \nabla \bar{U} \cdot \nabla F+\chi[u(u-\lambda v)-\bar{u}(\bar{u}-\underline{u})]$

$$
+\chi u(f-\lambda F)-\chi \bar{u}(\bar{f}-\underline{f})+g(u)-g(\bar{u})-a_{2}\left(u \int_{\Omega} u-\bar{u} \underline{u}\right)
$$

and this is equivalent to

$$
\begin{aligned}
\bar{U}_{t}-\Delta \bar{U}=- & \chi \nabla \bar{U} \cdot \nabla v+\bar{U}\left(\chi(u-\lambda v+\bar{u})+g^{\prime}(\xi)\right)+\chi \bar{u}(\underline{u}-\lambda v)-\chi \nabla \bar{U} \cdot \nabla F \\
& +\chi \bar{U}(f-\lambda F)+\chi \bar{u}(\bar{f}-\underline{f})+\chi \bar{u}(\underline{f}-\lambda F)-a_{2}\left(u \int_{\Omega} u-\bar{u} \underline{u}\right),
\end{aligned}
$$


where $\xi(x, t) \in(u(t, x), \bar{u}(t, x)) \cup(\bar{u}(t, x), u(t, x))$. We take $\bar{U}_{+}$as the test function in the previous equation, i.e. multiply by $\bar{U}_{+}$and integrate by parts over $\Omega$ to obtain, after some rutinary computations:

$$
\begin{aligned}
\frac{1}{2} \frac{\mathrm{d}}{\mathrm{d} t} \int_{\Omega} \bar{U}_{+}^{2}+ & \int_{\Omega}\left|\nabla \bar{U}_{+}\right|^{2}=\int_{\Omega} \bar{U}_{+}^{2}\left(\frac{\chi}{2}(u-\lambda v+2 \bar{u})+g^{\prime}(\xi)\right)+\int_{\Omega} \chi \bar{u}(\underline{u}-\lambda v) \bar{U}_{+} \\
& -\chi \int_{\Omega} \nabla \bar{U} \cdot \nabla F \bar{U}_{+}+\chi \int_{\Omega} \bar{U}(f-\lambda F) \bar{U}_{+}+\chi \int_{\Omega} \bar{u} \bar{U}_{+}(f-\bar{f}) \\
& +\chi \int_{\Omega} \bar{u} \bar{U}_{+}(\underline{f}-\lambda F)-a_{2} \int_{\Omega} \bar{U}_{+}\left(u \int_{\Omega} u-\bar{u} \underline{u}\right) .
\end{aligned}
$$

Since

$$
-\chi \int_{\Omega} \nabla \bar{U} \cdot \nabla F \bar{U}_{+}=\frac{\chi}{2} \int_{\Omega}(f-\lambda F) \bar{U}_{+}^{2}
$$

and

$$
\chi \int_{\Omega} \bar{u} \bar{U}_{+}(f-\bar{f}) \leqslant 0, \quad \chi \int_{\Omega} \bar{u} \bar{U}_{+}(\underline{f}-\lambda F) \leqslant 0
$$

it results

$$
\begin{aligned}
\frac{1}{2} \frac{\mathrm{d}}{\mathrm{d} t} \int_{\Omega} \bar{U}_{+}^{2}+ & \int_{\Omega}\left|\nabla \bar{U}_{+}\right|^{2} \leqslant \int_{\Omega} \bar{U}_{+}^{2}\left(\frac{\chi}{2}(u-\lambda v+2 \bar{u})+g^{\prime}(\xi)\right)+\int_{\Omega} \chi \bar{u}(\underline{u}-\lambda v) \bar{U}_{+} \\
& +\frac{3 \chi}{2} \int_{\Omega} \bar{U}_{+}^{2}(f-\lambda F)-a_{2} \int_{\Omega} \bar{U}_{+}\left(u \int_{\Omega} u-\bar{u} \underline{u}\right) .
\end{aligned}
$$

For the last two terms, after some computations, we obtain

$$
-\int_{\Omega} \bar{U}_{+}\left(u \int_{\Omega} u-\bar{u} \underline{u}\right)=-\int_{\Omega} \bar{U}_{+}^{2} \int_{\Omega} u-\bar{u} \int_{\Omega} \bar{U}_{+} \int_{\Omega}(u-\underline{u}) \leqslant \bar{u} \int_{\Omega} \bar{U}_{+} \int_{\Omega} \underline{U}-
$$

and

$$
\int_{\Omega} \bar{U}_{+}^{2}(f-\lambda F) \leqslant 2 C \int_{\Omega} \bar{U}_{+}^{2}
$$

The rest of the terms are treated as in the corresponding inequality in [26]. Then we have

$$
\frac{\mathrm{d}}{\mathrm{d} t} \frac{1}{2} \int_{\Omega} \bar{U}_{+}^{2}+\int_{\Omega}\left|\nabla \bar{U}_{+}\right|^{2} \leqslant k_{0} \int_{\Omega}\left(\bar{U}_{+}^{2}+\underline{U}_{-}^{2}\right)+\frac{k_{1}}{2} \int_{\Omega}(\underline{u}-\lambda v)_{+}^{2}
$$

for some positive constants $k_{0}$ and $k_{1}$. In the same fashion, we obtain

$$
\frac{\mathrm{d}}{\mathrm{d} t} \frac{1}{2} \int_{\Omega} \underline{U}_{-}^{2}+\int_{\Omega}\left|\nabla \underline{U}_{-}\right|^{2} \leqslant k_{2} \int_{\Omega}\left(\bar{U}_{+}^{2}+\underline{U}_{-}^{2}\right)+k_{3} \int_{\Omega}(\lambda v-\bar{u})_{+}^{2} .
$$

Since $-\Delta v+(\lambda v-\bar{u})=\bar{U}$ taking $(\lambda v-\bar{u})_{+}$as a test function it follows

$$
\frac{1}{\lambda} \int_{\Omega}\left|\nabla(\lambda v-\bar{u})_{+}\right|^{2}+\int_{\Omega}\left|(\lambda v-\bar{u})_{+}\right|^{2} \leqslant \int_{\Omega} \bar{U}_{+}(\lambda v-\bar{u})_{+},
$$

and by the Hölder inequality, it is equivalent to

$$
\frac{1}{\lambda} \int_{\Omega}\left|\nabla(\lambda v-\bar{u})_{+}\right|^{2}+\frac{1}{2} \int_{\Omega}\left|(\lambda v-\bar{u})_{+}\right|^{2} \leqslant \frac{1}{2} \int_{\Omega} \bar{U}_{+}^{2},
$$

which implies

$$
\int_{\Omega}\left|(\lambda v-\bar{u})_{+}\right|^{2} \leqslant \int_{\Omega} \bar{U}_{+}^{2} .
$$


In the same way, we have

$$
\int_{\Omega}\left|(\lambda v-\underline{u})_{-}\right|^{2} \leqslant \int_{\Omega} \underline{U}^{2} .
$$

By (1.17)-(1.20), it results

$$
\frac{\mathrm{d}}{\mathrm{d} t}\left(\frac{1}{2} \int_{\Omega} \bar{U}_{+}^{2}+\frac{1}{2} \int_{\Omega} \bar{U}_{-}^{2}\right) \leqslant K\left(\int_{\Omega} \bar{U}_{+}^{2}+\int_{\Omega} \bar{U}_{-}^{2}\right) .
$$

Using $\bar{U}_{+}=\underline{U}-\underline{U}_{-}=0$ for $t=0$, we may invoke Gronwall's Lemma to achieve

$$
\bar{U}_{+}=\underline{U}_{-}=0,
$$

which proves the lemma.

A Priori estimates for $a_{2} \leqslant 0$. In this case, as in section 1.1 , we denote by $(\bar{u}, \underline{u})=$ $(\bar{u}(t), \underline{u}(t))$ the solution of the associated ODEs system

$$
\begin{cases}\bar{u}^{\prime}=\bar{u}\left[\tilde{a}_{0}^{+}-\left(a_{1}+a_{2}-\chi\right) \bar{u}-\chi \underline{u}\right], & t>0, \\ \underline{u}^{\prime}=\underline{u}\left[\tilde{a}_{0}^{-}-\chi \bar{u}-\left(a_{1}+a_{2}-\chi\right) \underline{u}\right], & t>0,\end{cases}
$$

with non-negative initial data and preserve the hypothesis $\tilde{a}_{0}^{ \pm}$are continuous and bounded above and below for $t \geqslant t_{0}$ by positive constants.

Under assumption $a_{1}>2 \chi-a_{2}$, we have that (1.21) is a competitive Lotka-Volterra system and there exists a solution $\left(\bar{u}^{*}(t), \underline{u}^{*}(t)\right)$ of (1.21) such that the inequalities

$$
\begin{aligned}
& \frac{\tilde{a}_{0 L}^{+}\left(a_{1}+a_{2}-\chi\right)-\chi \tilde{a}_{0 M}^{-}}{\left(a_{1}+a_{2}-2 \chi\right)\left(a_{1}+a_{2}\right)} \equiv s_{1} \leqslant \bar{u}^{*}(t) \leqslant r_{1} \equiv \frac{\tilde{a}_{0 M}^{+}\left(a_{1}+a_{2}-\chi\right)-\chi \tilde{a}_{0 L}^{-}}{\left(a_{1}+a_{2}\right)\left(a_{1}-2 \chi-a_{2}\right)}, \\
& \frac{\tilde{a}_{0 L}^{-}\left(a_{1}+a_{2}-\chi\right)-\chi \tilde{a}_{0 M}^{+}}{\left(a_{1}+a_{2}\right)\left(a_{1}+a_{2}-2 \chi\right)} \equiv r_{2} \leqslant \underline{u}^{*}(t) \leqslant s_{2} \equiv \frac{\tilde{a}_{0 M}^{-}\left(a_{1}+a_{2}-\chi\right)-\chi \tilde{a}_{0 L}^{+}}{\left(a_{1}+a_{2}\right)\left(a_{1}+a_{2}-2 \chi\right)}
\end{aligned}
$$

hold for $t_{0} \leqslant t<\infty$.

For every solution $(\bar{u}(t), \underline{u}(t))$ to $(1.21)$ we have that $\bar{u}(t)-\bar{u}^{*}(t) \rightarrow 0$ and $\underline{u}(t)-\underline{u} \underline{u}^{*}(t) \rightarrow$ 0 as $t \rightarrow \infty$ (see [1] for details) and passing to limit in the above inequalities, we have

$$
\lim _{t \rightarrow \infty} \bar{u}^{*}(t)=\lim _{t \rightarrow \infty} \underline{u}^{*}(t)=u^{*}=\frac{a_{0}}{a_{1}+a_{2}}
$$

where $u^{*}$ is the equilibrium point defined in (1.9).

To apply the comparison method, we proceed as in the case $a_{2}>0$. Only the last term in (1.16) is changed by

$$
-a_{2}\left(u \int u-\bar{u}^{2}\right)
$$

Taking into account that $-a_{2} \geqslant 0$, we operate

$$
\int_{\Omega} \bar{U}_{+}\left(u \int_{\Omega} u-\bar{u}^{2}\right)=\int_{\Omega} \bar{U}_{+}^{2} \int_{\Omega} u+\bar{u} \int_{\Omega} \bar{U}_{+} \int_{\Omega} u-\bar{u}^{2} \int_{\Omega} \bar{U}_{+}
$$

and applying lemma 1.15 , we obtain

$$
-a_{2} \int_{\Omega} \bar{U}_{+}\left(u \int_{\Omega} u-\bar{u}^{2}\right) \leqslant k_{0}^{\prime} \int_{\Omega} \bar{U}_{+}^{2}
$$

for some positive constant $k_{0}^{\prime}$. For the rest of the terms we proceed as in case $a_{2}>0$ to come to

$$
\underline{u}<u<\bar{u} \text {. }
$$


Proof of theorem 0.1 under assumption (0.5). To prove $(0.8)$, we observe that lemma 1.7 entails

$$
\left\|u-\frac{a_{0}}{a_{1}+a_{2}}\right\|_{L^{\infty}(\Omega)} \rightarrow 0 \quad \text { as } t \rightarrow \infty
$$

Applying the maximum principle to the second equation in (1.1) we have

$$
\min _{x \in \bar{\Omega}} u(x, t) \leqslant \lambda v(x, t) \leqslant \max _{x \in \bar{\Omega}} u(x, t) \quad \forall t \in(0, \infty) ;
$$

taking $t \rightarrow \infty$ and using (1.23), we end up with

$$
\lim _{t \rightarrow \infty}\left\|\lambda v-\frac{a_{0}}{a_{1}+a_{2}}\right\|_{L^{\infty}(\Omega)}=0
$$

which completes the proof.

\section{A two-species chemotactic system}

In this section, we consider a system of three partial differential equations modelling the spatiotemporal behaviour of two competitive populations of biological species, both of which are attracted chemotactically by the same signal substance. More precisely, we consider the initialboundary value problem $(0.2)$. Recall that constants $\chi_{i}, a_{i}$ and $b_{i}$ for $i=0,1,2$ are positive, $a_{i} \in \mathbb{R}, b_{i} \in \mathbb{R}$ for $i=3,4, f$ is uniformly bounded and $f \in C_{x, t}^{\alpha, \beta}(\bar{\Omega} \times[0, T])$ for $\beta \geqslant 1+\frac{\alpha}{2}$. In [28], the authors have considered the case $f=0, k_{1}=k_{2}=1, a_{i}=b_{i}=0$ (for $i=3,4$ ) and they have obtained that the system possesses a uniquely determined spatially homogeneous positive equilibrium $\left(u^{*}, v^{*}\right)$, globally asymptotically stable within a certain non-empty range of the logistic growth coefficients under some restriction between chemotaxis and logistic coefficients.

It is the goal of this section to investigate how far the latter result on global asymptotic stability of spatially uniform equilibria remains true in the two-species system $(0.2)$. In order to prove theorem 0.2 , we specify the precise mathematical setting.

We denote by $W:=w-F$ where $F$ is the solution of $-\Delta F+F=f$, we can rewrite system (0.2) as follows:

$$
\left\{\begin{array}{l}
u_{t}-\Delta u=-\chi_{1} \nabla \cdot u(\nabla W+\nabla F)+u\left(a_{0}-a_{1} u-a_{2} v-a_{3} \int_{\Omega} u-a_{4} \int_{\Omega} v\right), \\
v_{t}-\Delta v=-\chi_{2} \nabla \cdot v(\nabla W+\nabla F)+v\left(b_{0}-b_{1} u-b_{2} v-b_{3} \int_{\Omega} u-b_{4} \int_{\Omega} v\right), \\
-\Delta W+\lambda W=k_{1} u+k_{2} v, \\
-\Delta F+\lambda F=f .
\end{array}\right.
$$

As a preliminary, as in the first problem studied in section 1, let us state the following result on local existence and uniqueness of solutions which can be proved by a straightforward adaptation of well-established methods (see for instance [28, lemma 2.1], or [15]).

Lemma 2.1. Let $\lambda, \chi_{i}, k_{i}$ be positive, $a_{i} \geqslant 0$ as well as $b_{i} \geqslant 0$ for $i=0,1,2$ and $a_{i} \in \mathbb{R}$, $b_{i} \in \mathbb{R}$ for $i=3,4$. We assume that $u_{0}$ and $v_{0}$ belong to $C_{x}^{\alpha}(\bar{\Omega})$, are non-negative and satisfy the boundary condition $\left(\partial u_{0} / \partial n\right)=\left(\partial v_{0} / \partial n\right)=0$. Then, there exist $T_{\max } \in(0, \infty]$ and $a$ unique triple $(u, v, w)$ of non-negative functions belonging to $C_{x, t}^{2+\alpha, 1+\frac{\alpha}{2}}\left(\bar{\Omega} \times\left(0, T_{\max }\right)\right)$ which solves $(0.2)-(0.4)$ in the classical sense in $\Omega \times\left(0, T_{\max }\right)$. Moreover,

either $T_{\max }=\infty \quad$ or $\quad\|u(\cdot, t)\|_{L^{\infty}(\Omega)}+\|v(\cdot, t)\|_{L^{\infty}(\Omega)} \rightarrow \infty \quad$ as $t \nearrow T_{\max }$. 


\subsection{A priori estimates and comparison method}

In this section, we consider the case $a_{i}>0, b_{i}>0$, for all $i=1, \ldots, 4$. As in previous section it is easy to prove that all the results are true for the cases where some or all coefficients $a_{3}, a_{4}, b_{3}, b_{4}$ are negative.

We analyse the ODEs system

$\bar{u}^{\prime}=\bar{u}\left[a_{0}+\chi_{1}(\bar{f}-\underline{f})-\left(a_{1}-k_{1} \chi_{1}\right) \bar{u}-\left(k_{1} \chi_{1}+a_{3}\right) \underline{u}+k_{2} \chi_{1} \bar{v}-\left(k_{2} \chi_{1}+a_{2}+a_{4}\right) \underline{v}\right]$,
$\underline{u}^{\prime}=\underline{u}\left[a_{0}+\chi_{1}(\underline{f}-\bar{f})-\left(k_{1} \chi_{1}+a_{3}\right) \bar{u}-\left(a_{1}-k_{1} \chi_{1}\right) \underline{u}-\left(k_{2} \chi_{1}+a_{2}+a_{4}\right) \bar{v}+k_{2} \chi_{1} \underline{v}\right]$,
$\bar{v}^{\prime}=\bar{v}\left[b_{0}+\chi_{2}(\bar{f}-\underline{f})+k_{1} \chi_{2} \bar{u}-\left(k_{1} \chi_{2}+b_{1}+b_{3}\right) \underline{u}-\left(b_{2}-k_{2} \chi_{2}\right) \bar{v}-\left(k_{2} \chi_{2}+b_{4}\right) \underline{v}\right]$,
$\underline{v}^{\prime}=\underline{v}\left[b_{0}+\chi_{2}(\underline{f}-\bar{f})-\left(k_{1} \chi_{2}+b_{1}+b_{3}\right) \bar{u}+k_{1} \chi_{2} \underline{u}-\left(k_{2} \chi_{2}+b_{4}\right) \bar{v}-\left(b_{2}-k_{2} \chi_{2}\right) \underline{v}\right]$,

for $t>0$, with initial conditions

$\bar{u}(0)=\bar{u}_{0}, \quad \underline{u}(0)=\underline{u}_{0}, \quad \bar{v}(0)=\bar{v}_{0} \quad$ and $\quad \underline{v}(0)=\underline{v}_{0}$.

The solutions to (2.3) are used as lower and upper solutions for the comparison method in lemma 2.3.

For any given initial data, there exists $T_{\max }=T_{\max }\left(\bar{u}_{0}, \underline{u}_{0}, \bar{v}_{0}, \underline{v}_{0}\right) \in(0, \infty]$ and a unique solution $(\bar{u}, \underline{u}, \bar{v}, \underline{v})$ of (2.3)-(2.4) in $\left(0, T_{\max }\right)$ such that it does not exist beyond $t=T_{\max }$. A straightforward computation shows that, for $\bar{f}-f=0$, there exists a constant steady state $\left(u^{*}, v^{*}\right)$ of $(0.2)$ defined in $(0.12)$ and $(0.13)$.

Note that $\left(u^{*}, v^{*}\right)$ determines a constant equilibrium of (2.3) for $\bar{f}-\underline{f}=0$, in the sense that

$$
\bar{u}^{*}=\underline{u}^{*}=u^{*}, \quad \bar{v}^{*}=\underline{v}^{*}=v^{*}
$$

defines a non-trivial data $\left(\bar{u}^{*}, \underline{u}^{*}, \bar{v}^{*}, \underline{v}^{*}\right)$ of system (2.3) which has attractivity property, as we shall see later. In this section, we study the convergence of solutions to the homogeneous steady state (see theorem 0.2 ). We prove, in lemma 2.2 that under assumptions

$$
0<\underline{u}_{0}<\underline{u}^{*}=\bar{u}^{*}<\bar{u}_{0} \quad \text { and } \quad 0<\underline{v}_{0}<\underline{v}^{*}=\bar{v}^{*}<\bar{v}_{0},
$$

the solution to (2.3)-(2.4) exists globally in time and converges to $\left(\bar{u}^{*}, \underline{u}^{*}, \bar{v}^{*}, \underline{v}^{*}\right)$ as $t$ goes to infinity.

Taking into account the results obtained in [28] where (0.2) and (2.3) were studied for $f=0$ and $a_{i}=b_{i}=0$ (for $i=3,4$ ), we are able to formulate the following result:

Lemma 2.2. Assume (0.5), (0.9)-(0.6) and let (2.6) hold. The solution $(\underline{u}, \bar{u}, \underline{v}, \bar{v})$ of (2.3) has the following properties:

(i) $\quad 0<\underline{u}(t)<\bar{u}(t)$ and $0<\underline{v}(t)<\bar{v}(t)$ for $t \in\left(0, \hat{T}_{\max }\right)$.

(ii) There exists $C<\infty$ such that we have

$$
\bar{u} \leqslant C \text { and } \bar{v} \leqslant C \quad \text { in }[0, \infty) \text {. }
$$

(iii)

$$
\bar{u}>\bar{u}^{*}=\underline{u}^{*}>\underline{u} \quad \text { and } \quad \bar{v}>\bar{v}^{*}=\underline{v}^{*}>\underline{v} \quad \text { in }[0, \infty),
$$

where $\left(\bar{u}^{*}, \underline{u}^{*}, \bar{v}^{*}, \underline{v}^{*}\right)$ denotes the equilibrium defined in (2.5).

(iv) Whenever the solution of (2.3)-(2.4) will be global in time and stabilize toward $\left(\bar{u}^{*}, \underline{u}^{*}, \bar{v}^{*}, \underline{v}^{*}\right)$ in the large time limit, i.e.

$$
\begin{aligned}
& \bar{u}(t) \rightarrow u^{*} \quad \text { and } \quad \underline{u}(t) \rightarrow u^{*} \quad \text { as } t \rightarrow \infty \quad \text { as well as } \\
& \bar{v}(t) \rightarrow v^{*} \quad \text { and } \quad \underline{v}(t) \rightarrow v^{*} \quad \text { as } t \rightarrow \infty,
\end{aligned}
$$

where $u^{*}$ and $v^{*}$ are as given by $(0.12),(0.13)$. 
Proof. The proof of (i), (ii) and (iii) is similar to the proof of lemmas 1.1-1.4 in [28] and the different terms of (2.3) do not change the solution behaviour.

We now give a sketch of the proof of part (iv) where hypothesis (0.6) plays an important role. We divide the first equation in (2.3) by $\bar{u}$ and the second by $\underline{u}$ to obtain, after subtraction, that

$$
\begin{aligned}
\frac{\mathrm{d}}{\mathrm{d} t} \log \frac{\bar{u}}{\underline{u}}=\frac{\bar{u}_{t}}{\bar{u}}-\frac{\underline{u}_{t}}{\underline{u}} \\
\quad=2 \chi_{1}(\bar{f}-\underline{f})-\left(a_{1}-2 k_{1} \chi_{1}-a_{3}\right)(\bar{u}-\underline{u})+\left(2 k_{2} \chi_{1}+a_{2}+a_{4}\right)(\bar{v}-\underline{v}) .
\end{aligned}
$$

As before, we have

$\frac{\mathrm{d}}{\mathrm{d} t} \log \frac{\bar{v}}{\underline{v}}=2 \chi_{2}(\bar{f}-\underline{f})+\left(2 k_{1} \chi_{2}+b_{1}+b_{3}\right)(\bar{u}-\underline{u})-\left(b_{2}+2 k_{2} \chi_{2}+b_{4}\right)(\bar{v}-\underline{v})$.

Adding the last two identities, we obtain

$$
\begin{gathered}
\frac{\mathrm{d}}{\mathrm{d} t}\left(\log \frac{\bar{u}}{\underline{u}}+\log \frac{\bar{v}}{\underline{v}}\right)=2\left(\chi_{1}+\chi_{2}\right)(\bar{f}-\underline{f})+\left(-a_{1}+2 k_{1}\left(\chi_{1}+\chi_{2}\right)+b_{1}+b_{3}+a_{3}\right)(\bar{u}-\underline{u}) \\
+\left(-b_{2}+2 k_{2}\left(\chi_{1}+\chi_{2}\right)+a_{2}+a_{4}+b_{4}\right)(\bar{v}-\underline{v})
\end{gathered}
$$

for all $t>0$. We denote by

$\epsilon_{0}:=\min \left\{a_{1}-2 k_{1}\left(\chi_{1}+\chi_{2}\right)-b_{1}-b_{3}-a_{3}, b_{2}-2 k_{2}\left(\chi_{1}+\chi_{2}\right)-a_{2}-a_{4}-b_{4}\right\}$

and the inequality becomes

$$
\frac{\mathrm{d}}{\mathrm{d} t}\left(\log \frac{\bar{u}}{\underline{u}}+\log \frac{\bar{v}}{\underline{v}}\right)=2\left(\chi_{1}+\chi_{2}\right)(\bar{f}-\underline{f})-\epsilon_{0}((\bar{u}-\underline{u})+(\bar{v}-\underline{v}))
$$

for all $t>0$.

By integration in (2.12) over $(0, t)$, thanks to assumptions $(0.9),(0.10),(0.6)$ and part $(i)$ of this lemma, we have

$$
\log \frac{\bar{u}}{\underline{u}}+\log \frac{\bar{v}}{\underline{v}} \leqslant \log \frac{\bar{u}_{0}}{\underline{u}_{0}}+\log \frac{\bar{v}_{0}}{\underline{v}_{0}}+2\left(\chi_{1}+\chi_{2}\right) \int_{0}^{\infty}(\bar{f}-\underline{f}):=C
$$

for all $t>0$. Using (2.9) it results

$$
\underline{u} \geqslant \frac{u^{*}}{C}>0, \quad \underline{v} \geqslant \frac{v^{*}}{C}>0
$$

By the mean value theorem we have

$$
\bar{u}(t)-\underline{u}(t)=\mathrm{e}^{\xi_{1}(t)}(\log \bar{u}(t)-\log \underline{u}(t))
$$

and

$$
\bar{v}(t)-\underline{v}(t)=\mathrm{e}^{\xi_{2}(t)}(\log \bar{v}(t)-\log \underline{v}(t))
$$

for some $\xi_{1}(t) \in(\log \underline{u}(t), \log \bar{u}(t))$ and $\xi_{2}(t) \in(\log \underline{v}(t), \log \bar{v}(t))$. By (2.13) we can rewrite (2.11)

$\frac{\mathrm{d}}{\mathrm{d} t}\left(\log \frac{\bar{u}}{\underline{u}}+\log \frac{\bar{v}}{\underline{v}}\right) \leqslant 2\left(\chi_{1}+\chi_{2}\right)(\bar{f}-\underline{f})-\epsilon_{1}\left(\log \frac{\bar{u}}{\underline{u}}+\log \frac{\bar{v}}{\underline{v}}\right) \quad$ for all $t>0$,

for some $\epsilon_{1}>0$. 
We solve the previous inequality to show that

$$
\begin{aligned}
\log \frac{\bar{u}}{\underline{u}}+\log \frac{\bar{v}}{\underline{v}} & \leqslant\left(\log \frac{\bar{u}_{0}}{\underline{u}_{0}}+\log \frac{\bar{v}_{0}}{\underline{v}_{0}}\right) \mathrm{e}^{-\epsilon_{1} t}+2\left(\chi_{1}+\chi_{2}\right) \int_{0}^{t} \mathrm{e}^{-\epsilon_{1}(t-s)}(\bar{f}-\underline{f}) \mathrm{d} s \\
& \text { for all } t>0 .
\end{aligned}
$$

Note that for any $\epsilon_{1}>0$, as a consequence of (0.6), the following limit holds

$$
\int_{0}^{t} \mathrm{e}^{-\epsilon_{1}(t-s)}\left|\sup _{x \in \Omega} f-\inf _{x \in \Omega} f\right| \mathrm{d} s \rightarrow 0 \quad \text { as } \quad t \rightarrow \infty .
$$

Then, thanks to (2.13) we conclude

$$
|\bar{u}(t)-\underline{u}(t)|+|\bar{v}(t)-\underline{v}(t)| \rightarrow 0 \quad \text { as } t \rightarrow \infty .
$$

As in the above section, thanks to strong maximum principle (see [24, proposition 52.7 , p 511]) we may assume that

$$
u_{0}>0 \quad \text { and } \quad v_{0}>0 \quad \text { in } \bar{\Omega} .
$$

In that point of the proof we need to prove that the solutions are bounded by the solutions of the ODEs system, i.e. the inequalities $\underline{u} \leqslant u \leqslant \bar{u}$ and $\underline{v} \leqslant v \leqslant \bar{v}$ in $\Omega \times\left(0, T_{\max }\right)$ hold.

Lemma 2.3. The solution of (0.2)-(0.4) satisfies

$$
\underline{u}(t) \leqslant u(x, t) \leqslant \bar{u}(t) \quad \text { for all } x \in \Omega \text { and } t \in\left(0, T_{\max }\right)
$$

and

$$
\underline{v}(t) \leqslant v(x, t) \leqslant \bar{v}(t) \quad \text { for all } x \in \Omega \text { and } t \in\left(0, T_{\max }\right),
$$

where $\underline{u}, \bar{u}, \underline{v}$ and $\bar{v}$ are as specified above.

Proof. As the proof of the corresponding comparison result for one species, we introduce the following functions

$$
\bar{U}(x, t):=u(x, t)-\bar{u}(t), \quad \underline{U}(x, t):=u(x, t)-\underline{u}(t)
$$

and

$$
\bar{V}(x, t):=v(x, t)-\bar{v}(t), \quad \underline{V}(x, t):=v(x, t)-\underline{v}(t)
$$

for $(x, t) \in \Omega \times\left[0, T_{\max }\right)$.

Note that for any $T<T_{\max }$ as a consequence of the regularity of the solutions $(u, v, w)$ we have

$u(x, t) \leqslant c_{1}(T), \quad v(x, t) \leqslant c_{1}(T) \quad$ and $\quad w(x, t) \leqslant c_{1}(T) \quad$ in $\Omega \times(0, T)$.

From (2.1), we obtain

$$
\begin{aligned}
u_{t}-\Delta u+\chi_{1} \nabla & u \nabla W+\chi_{1} \nabla u \nabla F=\chi_{1} u\left(k_{1} u+k_{2} v-\lambda W\right)+\chi_{1} u(f-\lambda F) \\
+ & u\left(a_{0}-a_{1} u-a_{2} v-a_{3} \int_{\Omega} u-a_{4} \int_{\Omega} v\right), \quad \text { in } \Omega \times\left(0, T_{\max }\right) .
\end{aligned}
$$


Then, thanks to the first ODE in (2.3), $\bar{U}$ satisfies

$$
\begin{aligned}
\bar{U}_{t}-\Delta \bar{U}+\chi_{1} & \nabla \bar{U} \cdot \nabla W+\chi_{1} \nabla \bar{U} \cdot \nabla F=\chi_{1} u(f-\lambda F) \\
& +u\left(a_{0}-a_{1} u-a_{2} v-a_{3} \int_{\Omega} u-a_{4} \int_{\Omega} v\right) \\
& +\chi_{1} u\left(k_{1} u+k_{2} v-\lambda W\right) \\
& -\bar{u}\left[a_{0}+\chi_{1}(\bar{f}-\underline{f})-\left(a_{1}-k_{1} \chi_{1}\right) \bar{u}-\left(k_{1} \chi_{1}+a_{3}\right) \underline{u}\right. \\
& \left.+k_{2} \chi_{1} \bar{v}-\left(k_{2} \chi_{1}+a_{2}+a_{4}\right) \underline{v}\right] \\
& =\mu_{1} \bar{U}+u\left[\left(\chi_{1}-\mu_{1}\right) u+\left(\chi_{1}-\mu_{1} a_{1}\right) v-\lambda \chi_{1} W\right] \\
& -\bar{u}\left[\left(\chi_{1}-\mu_{1}\right) \bar{u}-\chi_{1} \underline{u}+\chi_{1} \bar{v}-\left(\chi_{1}+\mu_{1} a_{1}\right) \underline{v}\right]
\end{aligned}
$$

in $\Omega \times\left(0, T_{\max }\right)$.

We multiply the previous equation by $\bar{U}_{+}$and after integration it follows

$$
\begin{aligned}
\frac{\mathrm{d}}{\mathrm{d} t} \frac{1}{2} \int_{\Omega} \bar{U}_{+}^{2}+ & \int_{\Omega}\left|\nabla \bar{U}_{+}\right|^{2}=-\chi_{1} \int_{\Omega} \bar{U}_{+} \nabla \bar{U} \cdot \nabla W+\int_{\Omega} \bar{U}_{+}\left(u\left(a_{0}-a_{1} u-a_{2} v\right)\right. \\
& +\chi_{1} \int_{\Omega} \bar{U}_{+} u\left(k_{1} u+k_{2} v-\lambda w\right)-\int_{\Omega} \bar{U}_{+} \bar{u} \\
& \times\left(a_{0}-\left(a_{1}-k_{1} \chi_{1}\right) \bar{u}-\left(k_{1} \chi_{1}\right) \underline{u}+k_{2} \chi_{1} \bar{v}-\left(k_{2} \chi_{1}+a_{2}\right) \underline{v}\right) \\
& +\left[-\chi_{1} \int_{\Omega} \bar{U}_{+} \nabla \bar{U} \cdot \nabla F+\chi_{1} \int_{\Omega} \bar{U}_{+} u(f-\lambda F)-\int_{\Omega} \bar{U}_{+} u\left(a_{3} \int_{\Omega} u+a_{4} \int_{\Omega} v\right)\right. \\
& \left.+\int_{\Omega} \bar{U}_{+} \bar{u}\left(-\chi_{1}(\bar{f}-\underline{f})+a_{3} \underline{u}+a_{4} \underline{v}\right)\right] .
\end{aligned}
$$

Since the proof of the lemma is similar to the proof of lemma 2.3 in [28], we do not reproduce the terms treated there and we only give estimations and details for the following terms:

$$
\begin{aligned}
{\left[-\chi_{1} \int_{\Omega} \bar{U}_{+} \nabla \bar{U}\right.} & \cdot \nabla F+\chi_{1} \int_{\Omega} \bar{U}_{+} u(f-\lambda F)-\chi_{1} \bar{u} \int_{\Omega} \bar{U}_{+}(\bar{f}-\underline{f}) \\
& \left.-\int_{\Omega} \bar{U}_{+} u\left(a_{3} \int_{\Omega} u+a_{4} \int_{\Omega} v\right)+\int_{\Omega} \bar{U}_{+} \bar{u}\left(a_{3} \underline{u}+a_{4} \underline{v}\right)\right] .
\end{aligned}
$$

Moreover, except the integrals

$$
-a_{4} \int_{\Omega} \bar{U}_{+} u \int_{\Omega} v+a_{4} \int_{\Omega} \bar{U}_{+} \bar{u} \underline{v},
$$

the rest of the terms have been treated in (1.7). Since

$$
-a_{4} \int_{\Omega} \bar{U}_{+} u \int_{\Omega} v+a_{4} \int_{\Omega} \bar{U}_{+} \bar{u} \underline{v}=a_{4} \int_{\Omega} \bar{U}_{+}\left(-u \int_{\Omega} v+\bar{u} \underline{v}\right)
$$

and

$$
\left(-u \int_{\Omega} v+\bar{u} \underline{v}\right)=-\bar{U} \int_{\Omega} v+\bar{u}\left(\underline{v}-\int_{\Omega} v\right) \leqslant-\bar{U} \int_{\Omega} v+\bar{u}\left(\int_{\Omega} \underline{V}\right),
$$

we rewrite

$$
-a_{4} \int_{\Omega} \bar{U}_{+} u \int_{\Omega} v+a_{4} \int_{\Omega} \bar{U}_{+} \bar{u} \underline{v} \leqslant-a_{4} \int_{\Omega} v \int_{\Omega} \bar{U}_{+}^{2}+a_{4} \bar{u} \int_{\Omega} \bar{U}_{+} \int_{\Omega} \underline{V}
$$


Thanks to the above inequalities we have

$$
\frac{\mathrm{d}}{\mathrm{d} t} \frac{1}{2} \int_{\Omega} \bar{U}_{+}^{2}+\int_{\Omega}\left|\nabla \bar{U}_{+}\right|^{2} \leqslant k_{1}(T)\left(\int_{\Omega} \bar{U}_{+}^{2}+\int_{\Omega} \underline{U}_{-}^{2}+\int_{\Omega} \bar{V}_{+}^{2}+\int_{\Omega} \underline{V}_{-}^{2}\right)
$$

for all $t \in(0, T)$, where $k_{1}(T)$ is a positive constant. In the same fashion as before we derive

$\frac{\mathrm{d}}{\mathrm{d} t} \frac{1}{2} \int_{\Omega} \underline{U}_{-}^{2}+\int_{\Omega}\left|\nabla \underline{U}_{-}\right|^{2} \leqslant k_{2}(T)\left(\int_{\Omega} \bar{U}_{+}^{2}+\int_{\Omega} \underline{U}_{-}^{2}+\int_{\Omega} \bar{V}_{+}^{2}+\int_{\Omega} \underline{V}_{-}^{2}\right)$,

$\frac{\mathrm{d}}{\mathrm{d} t} \frac{1}{2} \int_{\Omega} \bar{V}_{+}^{2}+\int_{\Omega}\left|\nabla \bar{V}_{+}\right|^{2} \leqslant k_{3}(T)\left(\int_{\Omega} \bar{U}_{+}^{2}+\int_{\Omega} \underline{U}^{2}+\int_{\Omega} \bar{V}_{+}^{2}+\int_{\Omega} \underline{V}^{2}\right)$

and

$\frac{\mathrm{d}}{\mathrm{d} t} \frac{1}{2} \int_{\Omega} \underline{V}_{-}^{2}+\int_{\Omega}|\nabla \underline{V}|^{2} \leqslant k_{4}(T)\left(\int_{\Omega} \bar{U}_{+}^{2}+\int_{\Omega} \underline{U}_{-}^{2}+\int_{\Omega} \bar{V}_{+}^{2}+\int_{\Omega} \underline{V}^{2}\right)$.

We finally add (2.17)-(2.20) and apply Gronwall's lemma to see, thanks to the election of the initial data $\underline{u}_{0}, \bar{u}_{0}, \underline{v}_{0}$ and $\bar{v}_{0}$

$$
0<\underline{u}_{0} \leqslant u_{0} \leqslant \bar{u}_{0}, \quad 0<\underline{v}_{0} \leqslant v_{0} \leqslant \bar{v}_{0}
$$

that

$$
\int_{\Omega}\left(\bar{U}_{+}^{2}+\underline{U}_{-}^{2}+\bar{V}_{+}^{2}+\underline{V}_{+}^{2}\right)=0 \quad \text { for all } t \in(0, T)
$$

Now, since $T \in\left(0, T_{\max }\right)$ is arbitrary, the proof of the lemma ends.

The proof of theorem 0.2 is a consequence of lemmata 2.2 and 2.3 for the case

$$
a_{i}, b_{i} \geqslant 0 \quad \text { for } \quad i=1, \ldots, 4 \text {. }
$$

If (2.21) is not satisfied, the system of ordinary differential equations has to be modified as follows:

$$
\begin{aligned}
& \bar{u}^{\prime}=\bar{u}\left[A_{10}-A_{11} \bar{u}-A_{12} \underline{u}+A_{13} \bar{v}-A_{14} \underline{v}\right], \\
& \underline{u}^{\prime}=\underline{u}\left[A_{20}-A_{21} \bar{u}-A_{22} \underline{u}-A_{23} \bar{v}+A_{24} \underline{v}\right], \\
& \bar{v}^{\prime}=\bar{v}\left[B_{10}+B_{11} \bar{u}-B_{12} \underline{u}-B_{13} \bar{v}-B_{14} \underline{v}\right], \\
& \underline{v}^{\prime}=\underline{v}\left[B_{20}-B_{21} \bar{u}+B_{22} \underline{u}-B_{23} \bar{v}-B_{24} \underline{v}\right],
\end{aligned}
$$

for $t>0$, where

$$
\begin{array}{ll}
A_{10}=a_{0}+\chi_{1}(\bar{f}-\underline{f}) & A_{20}=a_{0}-\chi_{1}(\bar{f}-\underline{f}), \\
A_{11}=A_{22}=a_{1}-\overline{k_{1}} \chi_{1}-\left(a_{3}\right)_{-} & A_{12}=A_{21}=k_{1} \chi_{1}+\left(a_{3}\right)_{+}, \\
A_{13}=A_{24}=k_{2} \chi_{1}+\left(a_{4}\right)_{-} & A_{14}=A_{23}=k_{2} \chi_{1}+a_{2}+\left(a_{4}\right)_{+}, \\
B_{10}=B_{20}=b_{0}+\chi_{2}(\bar{f}-\underline{f}) & B_{20}=b_{0}-\chi_{2}(\bar{f}-\underline{f}) \\
B_{11}=B_{12}=k_{1} \chi_{2}+\left(b_{3}\right)_{-} & B_{12}=B_{21}=k_{1} \chi_{2}+b_{1}+\left(b_{3}\right)_{+}, \\
B_{13}=B_{24}=b_{2}-k_{2} \chi_{2}-\left(b_{4}\right)_{-} & B_{14}=B_{23}=k_{2} \chi_{2}+\left(b_{4}\right)_{+}
\end{array}
$$

with initial conditions

$$
\bar{u}(0)=\bar{u}_{0}, \quad \underline{u}(0)=\underline{u}_{0}, \quad \bar{v}(0)=\bar{v}_{0} \quad \text { and } \quad \underline{v}(0)=\underline{v}_{0} .
$$

The rest of the proof is similar to the case $a_{i}, b_{i}>0$.

Remark 2.4. The proof of theorem 0.1 under assumption $(0.6)$ is similar to the proof of the two-species problem, therefore we omit the details. 


\section{Conclusion and discussion}

We have considered two systems of reaction-diffusion equations coupled in the differentiated factors and containing non-local terms. The problems arise from biological and chemical processes modelled by parabolic-elliptic chemotactic systems of equations. We have applied a comparison method to obtain the asymptotic behaviour of the solutions by using a system of ordinary differential equations. We prove the results under hypotheses for the coefficient equations which essentially reflect the assumption that the effects stemming from chemotactic cross-diffusion and competitive degradation are sufficiently small compared with the growth factors.

The novelty of the system consists of the combination of growth and forcing terms in chemotaxis systems. The first development in our study consists of these choices of growth and forcing terms. The first model $(0.1)$ introduces a growth term defined by a logistic function $g$ which contains a non-local term, frequently found in the literature. After normalization, $g$ has the following expression:

$$
g(u)=u\left(a_{0}-a_{1} u-a_{2} \int_{\Omega} u\right),
$$

with $a_{0}, a_{1}$ positive constants and $a_{2} \in \mathbb{R}$.

In the one-species case, concerning the associated ODEs system, we apply the existing results for Lotka-Volterra competition systems. Hypotheses $(0.5),(1.11)$ are essential to prove the existence of a unique positive stable equilibrium point $u^{*}$ of (1.8) given by (1.9), such that it globally attracts all solutions of $(0.1)$. If one of the conditions (1.11) fails, sometimes it is referred to as the principle of competitive exclusion, i.e. there can be no coexistence of the two species $\underline{u}$ and $\bar{u}$ in (1.5); one of them will be driven to extinction while the other will stabilize at a certain solution of a logistic equation. As we stated in the introduction, this case only provides information about the boundedness of solution of $(0.1)$ and no information about the asymptotic behaviour. So, assumptions (1.11) are essential to find optimal bounds for the general ODE system (1.5). Under restriction (0.5), both limits in (1.11) coincide and give us (1.10). A remaining problem concerning whether it is an optimum method is still open.

The second system, with two biological species, possesses a uniquely determined spatially homogeneous positive equilibrium $\left(u^{*}, v^{*}\right)$. The equilibrium is globally asymptotically stable within a certain non-empty range of the logistic growth coefficients under some restriction between chemotaxis and logistic coefficients. As in the first model, the hypothesis (0.6) ensures the global attractiveness of the steady state $\left(u^{*}, v^{*}\right)$. It guarantees the coexistence of the species under some restrictions in the parameters of the logistic term. To the authors' knowledge, the existing results in the literature for general systems of ODEs cannot be applied to prove the asymptotic stability of the second case.

The forcing term, $f$, is understood as a direct application of a chemical substance with implications on the behaviour of the solutions. A natural question and, for the moment, one mathematically interesting open problem is to understand if and how it is possible to control the populations controlling only the function $f$. Studying it could be worthwhile, because it might be thought of as a preparatory step for the corresponding optimal control problem which targets approaching a desired distribution of cells after a given time by suitably adjusting an external application of a signal in a small region of the domain.

\section{Acknowledgment}

This work is partly supported by project MTM2009-13655 MICINN (Spain). 


\section{References}

[1] Ahmad S 1987 Convergence and ultimate bound of solutions of the nonautonomous Volterra-Lotka competition equations J. Math. Anal. Appl. 127 377-87

[2] Ahmad S 1993 On the nonautonomous Volterra-Lotka competition equations Proc. Am. Math. Soc. 117 199-204

[3] Biler P 2008 Radially symmetric solutions of a chemotaxis model in the plane-the supercritical case Parabolic and Navier-Stokes Equations vol 81 (Banach Center Publications) pp 31-42

[4] Braun M 1983 Differential Equations and their Applications (New York: Springer)

[5] Conca $C$ and Espejo E 2012 Threshold condition for global existence and blow-up to a radially symmetric drift-diffusion system Appl. Math. Lett. $25352-6$

[6] Conca C, Espejo E and Vilches K 2011 Remarks on the blowup and global existence for a two species chemotactic Keller-Segel system in R2 Eur. J. Appl. Math. 22 553-80

[7] Espejo-Arenas E E, Stevens A and Velázquez J J L 2009 Simultaneous finite time blow-up in a two-species model for chemotaxis Analysis $29317-38$

[8] Espejo-Arenas E E, Stevens A and Velázquez J J L 2010 A note on non-simultaneous blow-up for a drift-diffusion model Diff. Integral Eqns 23 451-62

[9] Espejo-Arenas E E, Stevens A and Suzuki T 2011 Simultaneous blowup and mass separation during collapse in an interacting system of chemotactic species Diff. Integral Eqns 25 251-88

[10] Fasano A, Mancini A and Primicerio M 2004 Equilibrium of two populations subject to chemotaxis $M a t h$. Models Methods Appl. Sci. $14503-33$

[11] Herrero M A, Medina E and Velazquez J J L 1997 Finite-time aggregation into a single point in a reaction-diffusion system Nonlinearity 10 1739-54

[12] Hillen T and Painter K J 2009 A users guide to PDE models for chemotaxis J. Math. Biol. 58 183-217

[13] Horstmann D 2003 From 1970 until present: the Keller-Segel model in chemotaxis and its consequences: I. Jahresbericht Deutschen Mathematiker-Vereinigung 105 103-65

[14] Horstmann D 2011 Generalizing the Keller-Segel model: Lyapunov functionals, steady state analysis, and blow-up results for multi-species chemotaxis models in the presence of attraction and repulsion between competitive interacting species J. Nonlinear Sci. 21 231-70

[15] Horstmann D and Winkler M 2005 Boundedness vs. blow-up in a chemotaxis system J. Diff. Eqns 215 52-107

[16] Jäger W and Luckhaus S 1992 On explosions of solutions to a system of partial differential equations modeling chemotaxis Trans. Am. Math. Soc. 329 819-24

[17] Keller E F and Segel L A 1970 Initiation of slime mold aggregation viewed as an instability J. Theor. Biol. $26399-415$

[18] Keng D 2008 On a nonlocal reaction-diffusion population model Discrete Contin. Dyn. Syst. Ser. B $965-73$

[19] Kuiper H J 2001 A priori bounds and global existence for a strongly coupled quasilinear parabolic system modeling chemotaxis Electron J. Diff. Eqns $\mathbf{5 2} 18$

[20] Lauffenburger D A 1991 Quantitative studies of bacterial chemotaxis and microbial population dynamics Microbial. Ecol. 22 175-85

[21] Lebiedz D and Brandt-Pollmann U 2003 Manipulation of self-aggregation patterns and waves in a reaction-diffusion system by optimal boundary control strategies Phys. Rev. Lett. $91208-301$

[22] Nagai T 2001 Blowup of nonradial solutions to parabolic-elliptic systems modeling chemotaxis in twodimensional domains J. Inequal. Appl. $637-55$

[23] Negreanu M and Tello J I 2013 On a parabolic-elliptic chemotactic system with non-constant chemotactic sensitivity Nonlinear Anal.: Theory Methods and Appl. 80 1-13

[24] Quittner P and Souplet P 2007 Superlinear Parabolic Problems: Blow-up, Global Existence and Steady States (Birkhauser Advanced Texts) (Basel: Birkhauser)

[25] Szymánska Z, Morales Rodrigo C, Lachowicz M and Chaplain M A J 2009 Mathematical modelling of cancer invasion of tissue: the role and effect of nonlocal interaction Math. Models Methods Appl. Sci. 19 257-81

[26] Tello J I and Winkler M 2007 A chemotaxis system with logistic source Commun. Partial Diff. Eqns 32 49-877

[27] Tello J I and Winkler M 2013 Reduction of critical mass in a chemotaxis system by external application of chemoattractant Annali della Scuola Normale Superiore di Pisa submitted

[28] Tello J I and Winkler M2012 Stabilization in a two-species chemotaxis system with a logistic source Nonlinearity $251413-25$

[29] Wang X and Wu Y 2002 Qualitative analysis on a chemotactic diffusion model for two species competing for a limited resource Q. Appl. Math. 60 505-31

[30] Winkler M 2011 Blow-up in a higher-dimensional chemotaxis system despite logistic growth restriction J. Math. Anal. Appl. $384261-72$ 